\title{
Visual stimulation with food pictures in regulation of hunger hormones and nutrient deposition
}

\section{Abstract}

Food cues modulate hunger and dictate nutritional choices. The omnipresent stimulus of palatable food promotes the epidemics of obesity and metabolic diseases. The aim of our study was to investigate the impact of visual stimulation with food pictures on appetite-related hormones as well as the functionality of the secreted hormones and its impact on macronutrient uptake and deposition in healthy subjects. Additionally, we sought to identify differences in response to visual food cues of total and active ghrelin to and to a meal followed by the stimulation.

Healthy, non-obese participants were recruited for two two-day studies. During the first study, the subjects were shown random non-food pictures on the first day and pictures of appetizing foods on the second day of the study. The second study followed the same schedule and additionally, following the picture session, the participants were asked to drink a milkshake. The subjects reported subjective hunger. Levels of glucose, triglycerides and hunger-related hormones were measured in blood. In addition, an animal study was performed, during which 4 hours fasted mice were submitted to visual and olfactory stimulation using chow, while not being able to access the food. Following the stimulation, oral glucose tolerance test was performed, gene expression and blood total, as well as acylated ghrelin, were measured.

Exposure to food pictures affected participant's blood glucose and incretin levels. Concentrations of several hormones in the blood were interdependent and in case of ghrelin and GIP as well as ghrelin and GLP-1 this co-occurrence relied on the visual cues. Changes of total ghrelin concentration following food stimulation was highly individual and participants could be grouped as ghrelin responders or non-responders. The responders showed upregulated total ghrelin and their total ghrelin levels correlated with food picture score revealing that in ghrelin non-responders reaction to food pictures was diminished. The concentration of active ghrelin decreased following a meal but was not affected by exposure to the pictures of food. Protein content and color intensity of food pictures correlated reversely with participants' rating of the pictures. Sensory stimulation of mice resulted in increased blood glucose levels.

We conclude that observation of food pictures significantly influences blood glucose levels and concentration of several appetite-related hormones. The protein content of the observed foods and green color intensity in pictures may serve as a predictor of subjective attractiveness of the presented meal.

\section{Conflict of Interest}

There is no conflict of interest 